\title{
Discovery of A New Class of Pulsating Stars: Gravity-mode Pulsators among Subdwarf B Stars
}

\author{
E. M. Green ${ }^{1}$, G. Fontaine ${ }^{2}$, M. D. Reed ${ }^{3}$, K. Callerame ${ }^{1}$, I. R. Seitenzahl ${ }^{1}$, B. A. White ${ }^{1}$, \\ E. A. Hyde ${ }^{1}$, R. Østensen ${ }^{4}$, O. Cordes ${ }^{5}$, P. Brassard ${ }^{2}$, S. Falter ${ }^{6}$, E. J. Jeffery ${ }^{7}$, S. Dreizler ${ }^{8}$, \\ S. L. Schuh ${ }^{8}$, M. Giovanni ${ }^{1}$, H. Edelmann ${ }^{6}$, J. Rigby $^{1}$, and A. Bronowska ${ }^{4}$ \\ egreen@as.arizona.edu
}

\begin{abstract}
During the course of an ongoing CCD monitoring program to investigate low-level light variations in subdwarf B (sdB) stars, we have serendipitously discovered a new class of low amplitude, multimode sdB pulsators with periods of the order of an hour. These periods are more than a factor of ten longer than those of previously known multimode sdB pulsators (EC 14026 stars), implying that they are due to gravity modes rather than pressure modes. The longer period pulsators are found only among cooler sdB stars, where they are surprisingly common. The iron opacity instability that drives the short period EC 14026 stars is effective only in hot sdB's, leaving the driving mechanism for the deeper gravity modes in cool sdB's currently unknown. We present the first observational results for our newly identified sdB variables, and discuss possible implications.
\end{abstract}

\footnotetext{
${ }^{1}$ Steward Observatory, University of Arizona, Tucson, AZ 85721; egreen@as.arizona.edu; callerame@cox.net; irs@uchicago.edu; bwhite@as.arizona.edu; elainahyde@yahoo.com; jrigby@as.arizona.edu

${ }^{2}$ Département de Physique, Université de Montréal, CP6128, Station Centreville, Montréal, QC H3C 3J7, Canada; fontaine@astro.umontreal.ca; brassard@astro.umontreal.ca

${ }^{3}$ Physics, Astronomy, \& Material Science, SW Missouri State University, Springfield, MO 65804; mreed@sdbv.smsu.edu

${ }^{4}$ Isaac Newton Group of Telescopes, 37800 Santa Cruz de La Palma, Canary Islands, Spain; roy@ing.iac.es; bronka@tiger.chem.uw.edu.pl

${ }^{5}$ Sternwarte of the University Bonn, Auf dem Huegel 71, D-53123 Bonn, Germany; ocordes@astro.unibonn.de

${ }^{6}$ Dr.-Remeis-Sternwarte University Erlangen-Nürnberg, Sternwartstr. 7, D-96049 Bamberg, Germany; falter@sternwarte.uni-erlangen.de

${ }^{7}$ Brigham Young University, Provo, UT 84602; ejj9@email.byu.edu

${ }^{8}$ Institut für Astronomie und Astrophysik, Universität Tübingen, Sand 1, D-72076 Tübingen, Germany; dreizler@astro.uni-tuebingen.de; schuh@astro.uni-tuebingen.de
} 
Subject headings: stars: horizontal-branch — stars: individual (PG 1716+426)

— stars: interiors — stars: oscillations — subdwarfs

\section{Introduction}

Subdwarf B (sdB) stars are evolved hot stars (22,000 to 40,000 K) commonly found in the disk of our Galaxy (e.g. Saffer et al. 1994). They are widely believed to be core Heburning stars of $\sim 0.5 M_{\odot}$ on the extended horizontal branch (EHB) (Heber 1986; Saffer et al. 1994), having lost nearly all of their envelopes near the first red giant tip. The existence of multimode pulsators among sdB stars presents a powerful opportunity for further investigation of their structure using asteroseismological techniques. In addition to clarifying the uncertain evolutionary histories of the sdB stars themselves, the results will be relevant to the later evolution of the vast majority of normal stars. Buried under their huge envelopes, all low mass He-burning red giants have cores that are basically identical to sdB stars. It is our good fortune that the latter have shed all but a few wisps of their obscuring envelopes, and that some appear to pulsate with exactly the right complexity required for asteroseismology.

The first pulsating sdB stars were discovered only recently (Kilkenny et al. 1997). Commonly called EC 14026 stars, after the prototype ${ }^{9}$, they are multimode pulsators with typical periods of 100-250 s, and a total range of about 80-600 s. Independently and nearly simultaneously, their existence was predicted by Charpinet et al. $(1996,1997)$, due to the presence of low-order and low-degree radial and nonradial pressure modes (p-modes) excited by an iron opacity $\kappa$ mechanism in the thin diffusion-dominated envelopes. Additional EC 14026 stars were soon found by teams at the South African Astronomical Observatory (Kilkenny 2002, and references therein), the University of Montreal (Billères et al. 2002), and the Nordic Optical Telescope (NOT) (Silvotti et al. 2002, and references therein), bringing the current total to about 30. The justifiable excitement over these stars (Koen et al. 1998a; Fontaine et al. 1998; O’Donoghue et al. 1999; Charpinet, Fontaine, \& Brassard 2001; Kilkenny 2002) stems from the fact that stellar and atmospheric models for sdB stars appear to be sufficiently realistic for nonadiabatic calculations to be extremely successful in explaining their properties (Charpinet et al. 2001). In agreement with the theoretical results, EC 14026 variables are found primarily among the hotter sdB stars, clustering around $T_{\text {eff }} \sim 33,500 \mathrm{~K}$ and $\log g \sim 5.8$, with only a few low gravity examples at somewhat cooler temperatures. ${ }^{10}$ The

\footnotetext{
${ }^{9}$ EC 14026 stars are now officially V361 Hya stars.

${ }^{10}$ PG 1605+072 (Koen et al. 1998b); Feige 48 (Koen et al. 1998c); HS 2201+2610 (Østensen et al. 2001a); HS 0702+6043 (Dreizler et al. 2001)
} 
pulsation amplitudes are generally less than a few hundredths of a magnitude.

In this letter, we introduce another chapter in sdB asteroseismology: the discovery of a new class of multimode pulsators. The newly identified stars have apparent periods at least a factor of ten longer than those observed in EC 14026 stars, implying that they must be due to gravity modes (g-modes). The longer periods present both advantages and disadvantages for further observations. The most important gain may be the greatly increased range and number of suitable EHB candidates for asteroseismology. The following sections describe the observations and apparent trends identified in the data, discuss their implications for the unknown excitation mechanism, and summarize our conclusions. Subsequent papers will examine individual pulsators in more detail.

\section{Observational Characteristics}

The prototype long period sdB variable, $\mathrm{PG} 1716+426$, is an unremarkable faint blue star less than $1^{\circ}$ south of M92 in Hercules ( $V \simeq 13.97$, Wesemael et al. 1992). Its variability was first detected in 1999, though its nature wasn't fully appreciated for nearly two years. The discovery light curve (shown at the top of Figure 1) was taken amidst thin cirrus at the Steward $2.3 \mathrm{~m}$ telescope on Kitt Peak, during initial tests of a pilot program to monitor sdB binaries with large radial velocity variations. We were searching for eclipses by white dwarfs, ellipsoidal effects, and reflection effects, all of which have amplitudes less than a few hundredths of a magnitude and timescales longer than $250 \mathrm{~s}$. Except where noted, all data for this paper were obtained at the Arizona $2.3 \mathrm{~m}$ and $1.6 \mathrm{~m}$ telescopes on Kitt Peak and Mt. Bigelow using conventional 2K CCD's binned 3x3 (0.45"/pixel) to reduce the overhead time to $24 \mathrm{~s}$. Depending on the target, integration times of $15-60 \mathrm{~s}$ were chosen to maximize the counts and minimize photometric errors. Differential magnitudes were derived from aperture photometry of the $\mathrm{sdB}$ relative to reference stars of comparable magnitude within each frame.

Six additional light curves for PG 1716+426, 2-4 hours each during April-June 2000, showed persistently irregular variations in both amplitude and phase, that were not present in any of several nearby reference stars. Both low resolution (Saffer et al. 1994) and very high $\mathrm{S} / \mathrm{N} 1 \AA$ resolution MMT spectra (Green et al., in preparation), and multicolor photometry (Wesemael et al. 1992) proved that PG $1716+426$ is a normal, moderately cool sdB star, but the timescales seemed much too long for an EC 14026 star. Eventually we detected similar variations in a second sdB, PG0101+039, although at lower amplitude. We were allocated 18 nights at Mt. Bigelow to monitor PG 0101+039, but were able to observe for only one night due to unseasonable bad weather. We finally acquired 81 hours of CCD photometry for 
PG 1716+426 during 14 nights in April-June 2001: 12 clear nights at the Mt. Bigelow $1.6 \mathrm{~m}$ telescope, several adjoining hours at the end of a partially cloudy night on the Calar Alto $2.2 \mathrm{~m}$, a separate night on the NOT, and another with the Fick Observatory $0.6 \mathrm{~m}$ telescope. At Mt. Bigelow and at Fick, all data were taken with an $R$ filter. The NOT observations cycled rapidly between $V, R$, and $I$ with ALFOSC (Østensen et al. 2001b), while the BUSCA camera at Calar Alto allowed simultaneous photometry in four wide passbands from $U$ to $I$ (Falter et al. 2003). A first analysis of all the $R$ data found at least 3-5 pulsation modes with periods between 0.8 and 1.4 hours. Further details will be presented by Reed et al. (in preparation).

Once pulsations were confirmed in PG 1716+426, we broadened the $1.6 \mathrm{~m}$ monitoring program to include a nearly random sample of bright $\operatorname{sdB}$ stars $(V \leq 14)$. We have now identified 20 stars (including PG 1716+426) that appear to be multimode pulsators with periods of the order of an hour, from a total sample of 72. Each star was monitored for 2 to 7 hours at a time, and most were observed on multiple nights to allow for the possibility of occasional cancellation of modes. (PG 1716+426 exhibited a nearly flat light curve for nearly four hours once, in 14 nights of observation.) The peak-to-peak amplitudes in the light curves are comparable with those of most EC 14026 stars. The strongest new pulsators clearly show the characteristic beating effects of multiple modes with close periods (Fig. 1). However, about half of our variables pulsate so weakly that relative light variations are detectable only because of the stability, precision, and good sky coverage of modern CCD's. We classify the weakest pulsators as bonafide members of the class only when light variations are clearly detected on more than one night, but even in their "low" states, the light curves are usually not as flat as those of non-pulsators. Daily aliasing is obviously a major problem, since fewer than 8 to 10 cycles per night can be observed from a single site. Extensive, well-coordinated multi-site campaigns or space observations over fairly long time baselines are required for good mode characterization.

The only selection biases arise from a preference for brighter targets, mostly from the PG catalog (Green, Schmidt, \& Liebert 1986), and our avoidance of sdB stars with composite spectra (for better temperature and gravity determinations). Otherwise, we selected stars having the best sky positions at the time of observation. We therefore expect our sample to be representative of all $\mathrm{sdB}$ stars for properties such as temperature, gravity, presence of close companions, etc. For example, PG $1716+426^{11}$ and the next few members of the class were all found in single-lined spectroscopic binaries with orbital periods of less than a few days, but this was due only to our pre-discovery sample selection. Now that we have

\footnotetext{
${ }^{11}$ We derive an orbital period of $1.77739^{\mathrm{d}}$ for PG $1716+426$; Morales-Rueda et al. (2002) found $1.77732^{\mathrm{d}}$.
} 
obtained light curves for most of a representative subsample of $52 \mathrm{sdB}$ stars for which we have precise radial velocities from multiple spectra, we find no binary correlation. Specifically, 29 of the $52 \mathrm{sdB}$ stars $(56 \%)$ are short period binaries, having the same apparent distribution in temperature and gravity as the rest of the sample. 13 of the 52 are also long period pulsators. If the pulsations are independent of close binary status, we expect $7.25 \pm 2.4$ binary pulsators. The observed number is 7 , indicating that the fraction of short period binaries among PG 1716-like stars is no different from that of sdB stars in general.

The present data suggest an upper limit of 90-120 minutes for the major periods of all the new pulsators. We have not found any sdB pulsators with obvious periods as long as the 7.8 hour sawtooth modulation detected by Orosz \& Wade (1999) in KPD 0422+5421, although we did find three low amplitude variables with periods of several hours or more. Two are sdB stars exhibiting apparent reflection effects, having appropriately phased sinusoidal light curves the same length as their orbital periods $\left(\sim 6^{\mathrm{h}}\right.$ and $\left.12^{\mathrm{h}}\right)$. The third was a reference star with an early $\mathrm{F}$ spectrum and a $6^{\mathrm{h}}$ period, a likely $\delta$ Scuti star.

A general characteristic of nonradial pulsators is the expected amplitude dependence of the pulsations as a function of wavelength, although the largest effects are in the ultraviolet (e.g. Robinson et al. 1995; Brassard, Fontaine, \& Wesemael 1995). Multicolor amplitudes have been reported for two short period (p-mode) pulsators. Koen (1998) observed somewhat larger $U$ amplitudes in KPD 2109+4401, but no significant trends in $B V R$. Falter et al. (2003) found similar behavior in PG 1605+072. Following Brassard et al. (1995), we computed the wavelength dependence of the amplitude of a g-mode in a $30,000 \mathrm{~K}, \log g=$ $5.5 \mathrm{sdB}$ model. We find that the theoretical curve is rather flat in the red and increases somewhat toward the blue: predicted amplitude ratios are 1.09 for $B / V, 1.38$ for $U / V$. Our observations appear mostly consistent. The NOT data for PG $1716+426$ show no obvious systematic trends in $V R I$, and (non-simultaneous) $2.3 \mathrm{~m} B V R$ amplitudes are fairly comparable. At first glance, the largest amplitude in the short and somewhat noisier BUSCA data appears to be in the $I$ filter, but closer inspection reveals that the amplitudes cannot be disentangled from surprisingly large slopes in the baselines (mainly in the wide $U$ and $I$ filters). Subsequent experiments with simultaneous $B$ and $R$ photometry in Arizona demonstrated that the baseline trends are almost certainly due to differential extinction between the sdB and the much redder comparison stars. With hour long timescales, there are serious problems at bluer wavelengths or with wider bandpasses, because the effect of differential extinction is comparable to the pulsational amplitudes. At Mt. Bigelow and Kitt Peak, we generally see significant differential extinction in $B$, a less noticeable effect in $V$, and no detectable effect in the (preferred) $R$ filter ( $I$ is usually avoided due to fringing).

Figure 2 compares the positions of the long period and short period sdB pulsators in 
the $\log g$ vs $T_{\text {eff }}$ diagram. The 13 newly identified pulsators (red and green error bars) for which we derived homogeneous temperatures and gravities are all cooler than 30,000 K, in marked contrast with the hotter EC 14026 stars (blue filled circles). We lack spectra for seven (unplotted) new pulsators, but six of those with temperatures from other sources also appear to be relatively cool (Heber 1986; Wesemael et al. 1992; Saffer et al. 1994; MoralesRueda et al. 2002). At first glance, there seems to be little overlap in temperature and gravity between the long and short period pulsators, and no long period variations have been found in any EC 14026 stars $^{12}$. However, we note that the inhomogeneous atmospheric parameters for the latter (Charpinet 2001) are not necessarily on the same scale as our data points. Our temperatures are systematically somewhat hotter than those derived by some other investigators for $T_{\text {eff }}<32,000 \mathrm{~K}$, and our gravities consequently slightly higher. (We are continuing to pursue the longstanding disagreement between various atmospheric codes for sdB stars (Wesemael et al. 1997), but it is beyond the scope of this paper.) Thus, it is probably premature to speculate about the (small) degree of separation or overlap between the two types of pulsators in Figure 2. The important point is that long period sdB pulsators clearly populate a separate temperature and gravity regime than do short period pulsators, in addition to their very different timescales.

The fraction of sdB stars exhibiting long period light variations is surprisingly high, in further contrast with the EC 14026 stars: roughly $75 \%$ of sdB stars cooler than $30,000 \mathrm{~K}$, or $25-30 \%$ of all sdB stars.

\section{Discussion}

Observed periods of the order of an hour require the newly discovered sdB variables to be gravity mode pulsators. Preliminary calculations show that high radial order g-modes are needed to produce the range of periods observed in PG 1716+426. The $\kappa$ mechanism that has been so successful for understanding p-mode pulsations in EC 14026 stars (Charpinet et al. 1997; Brassard et al. 2001) does not appear to be capable of driving such deep pulsational modes. The g-mode driving mechanism is therefore unknown.

The limited temperature range of the long period variables in Figure 2 implies the pulsation mechanism is intrinsic to the star, rather than an external driver such as tidal

\footnotetext{
${ }^{12}$ This is a weak constraint, since only a few EC 14026 stars are bright enough for our survey, and several of these have such complicated light curves that it would be very difficult to detect additional long period components, e.g. PG 1605+072 (extreme amplitudes), PG 1336-018 (eclipsing sdB + dM), or KPD 1930+2752 ( $\mathrm{sdB}+$ wd with ellipsoidal variations).
} 
excitation (Fontaine et al. 2003). The lower temperatures result from increased hydrogen envelope masses, up to $0.003-0.004 M_{\odot}$ in the coolest sdB stars. However, it is not clear how such apparently negligible envelopes could cause longer period pulsations.

Canonical EHB evolutionary sequences (e.g. Dorman's tracks, from Charpinet et al. (2000), shown in Figure 2) indicate that a star initially expands away from the zero age EHB, and then begins contracting toward higher temperatures as helium is consumed in the core. Following central helium exhaustion (where the slope of the tracks changes sign for the second time, very abruptly), helium shell burning produces a feeble attempt at further expansion, which is quickly succeeded by rapid contraction to the white dwarf stage. Our data suggest that larger amplitude g-mode pulsators (in red) occur preferentially (only?) toward the end of core helium burning (after correcting for a small apparent offset by shifting the theoretical tracks down until their starting points form a lower envelope to all the cooler stars). If strong pulsators do not exist near the zero age EHB, it is tempting to think that they might be driven by a slowly reviving hydrogen burning shell. However, in standard EHB models, the miniscule envelope does not allow the hydrogen shell to "turn on" until after the star has contracted to a very hot white dwarf. Thus, if the hydrogen envelope is really of key importance in the driving mechanism, its role could be elucidated only with nonstandard models of sdB stars. It would be interesting to know if similar g-mode pulsations can occur in sdB stars whose spectra show little if any hydrogen: the He-sdB, or sdB:He4 stars (Jeffery et al. 1997, and references therein), if any exist with comparable temperatures and gravities.

P. Moskalik (private communication) pointed out that the distribution of short- and long-period sdB pulsators in the $\log g$ vs $T_{\text {eff }}$ diagram resembles the situation in main sequence B stars. In both cases, the hotter objects are low-order p-mode pulsators, while the cooler stars are high-order g-mode pulsators. Dziembowski, Moskalik, \& Pamyatnykh (1993) found that the same mechanism could drive both types of pulsation on the main sequence. Perhaps a variant of Charpinet et al.'s (1997) $\kappa$ mechanism could be found to work in the cooler sdB stars, though this remains to be seen.

\section{Summary and Conclusions}

We have so far identified 20 subdwarf B stars that appear to be multimode pulsators

on timescales of about an hour, more than a factor of ten longer than typical periods of the recently discovered EC 14026 pulsators. Observed peak-to-peak amplitudes are $\lesssim 0.05$ magnitudes. Due to the longer timescales, only the prototype, PG 1716+426, has been sufficiently well observed to detect individual modes. Our first attempt, with 81 hours monitoring spread over 2 months, indicates the presence of at least 3-5 probable modes with periods 
between 0.8 and 1.4 hours. All long period sdB pulsators with known, spectroscopically determined temperatures are relatively cool $\left(\mathrm{T}_{\text {eff }}<30,000 \mathrm{~K}\right)$, in contrast with the hotter EC 14026 stars. PG 1716 stars are also about six times more common.

Many of the newly identified variables are easily observed with small telescopes capable of precise differential photometry. Fast frame transfer CCD's are not required; conventional CCD's may have an advantage if their larger field of view permits a better selection of reference stars. Good mode characterization will require photometry over a much larger fraction of a day than can be obtained from a single site, in addition to long time baselines. With ground-based campaigns, differential extinction is a much worse problem for long period sdB pulsators than for the short period ones, but it can be minimized by using an $\mathrm{R}$ filter. PG 1716 stars are excellent candidates for observation from space, to eliminate both aliasing and atmospheric problems.

The long periods found in PG 1716+426, and strongly indicated in the light curves of nineteen other cool sdB variables, imply the detection of high radial order g-modes. The driving mechanism for g-modes in sdB stars is currently unknown. The distribution of the stars in the $\log g-T_{\text {eff }}$ plane suggests that their longer pulsations might be related to thicker hydrogen envelopes, but this is not easily understood from standard EHB models. Alternatively, some variant of the EC 14026 iron opacity mechanism might also be at work in cooler sdB stars, analogous to the situation in main sequence B stars (Dziembowski et al. 1993).

The relatively long pulsation timescales, the distinctly different temperature range, and the inability of the existing iron opacity mechanism to drive g-modes, all support the identification of the long period sdB stars as a separate class of pulsating variables, one with great potential to expand our understanding of evolved stars.

This investigation was made possible by NSF grants AST-9731655 and AST-0098699. We thank the Steward TAC for their generous time allocations, and P. Strittmatter for his continuing support of undergraduate research. We are grateful to the SO staff, especially E. Christensen, B. Wood, and G. Rosenbaum, for their patience with our lengthy calibrations, and all their other assistance. EMG thanks Bill Peters for many tangible and intangible contributions too numerous to mention.

\section{REFERENCES}

Billères, M., Fontaine, G., Brassard, P., Liebert, J. 2002, ApJ, 578, 515 
Brassard, P., Fontaine, G., Billères, M., Charpinet, S., Liebert, J., \& Saffer, R.A. 2001, ApJ, 563,1013

Brassard, P., Fontaine, G., Wesemael, F. 1995, ApJS, 96, 545

Charpinet, S. 2001, AN, 322, 387

Charpinet, S., Fontaine, G., \& Brassard, P. 2001, PASP, 113, 775

Charpinet, S., Fontaine, G., Brassard, P., Chayer, P., Rogers, F.J., Iglesias, C.A., \& Dorman, B. 1997, ApJ, 483, L123

Charpinet, S., Fontaine, G., Brassard, P., \& Dorman, B. 1996, ApJ, 471, L103

Charpinet, S., Fontaine, G., Brassard, P., \& Dorman, B. 2000, ApJS, 131, 223

Dreizler, S., Schuh, S.L., Deetjen, J.L., Edelmann, H., Heber, U. 2001, A\&A, 386, 249

Dziembowski, W.A., Moskalik, P., \& Pamyatnykh, A.A. 1993, MNRAS, 265, 588

Falter, S., Heber, U., Dreizler, S., Schuh, S.L., \& Cordes, O. 2003, in White Dwarfs, ed. D. de Martino, R. Silvotti, J.-E. Solheim, \& R. Kalytis (Dordrecht:Kluwer), in press

Fontaine, G., Brassard, P., Charpinet, S., Green, E.M., \& Willems, B. 2003, in Asteroseismology Across the HR Diagram, ed. M.J. Thompson, M.S. Cunha, \& M.J.P.F.G. Monteiro (Dordrecht:Kluwer), in press

Fontaine, G., Charpinet, S., Brassard, P., Chayer, P., Rogers, F.J., Iglesias, C.A., \& Dorman, B. 1998, in IAU Symp. 185, New Eyes Inside the Sun and Stars, ed. F. Deubner \& D.W. Kurtz (Dordrecht: Kluwer), 367

Green, R.F., Schmidt, M., \& Liebert, J. 1986, ApJS, 61, 305

Heber, U. 1986, A\&A, 155, 33

Jeffery, C.S., Drilling, J.S., Harrison, P.M., Heber, U., Moehler, S. 1997, A\&AS, 125, 501

Kilkenny, D. 2002, in IAU Coll. 185, Radial and Nonradial Pulsations as Probes of Stellar Physics, ed. C. Aerts, T.R. Bedding, \& J. Christensen-Dalsgaard (San Francisco:ASP), 356

Kilkenny, D., Koen, C., O’Donoghue, D., \& Stobie, R.S. 1997, MNRAS, 285, 640

Koen, C. 1998, MNRAS, 300, 567 
Koen, C., Kilkenny, D., O’Donoghue, D., \& Stobie, R.S. 1998a, in IAU Symp. 185, New Eyes Inside the Sun and Stars, ed. F. Deubner \& D.W. Kurtz (Dordrecht:Kluwer), 361

Koen, C., O’Donoghue, D., Kilkenny, D., Lynas-Gray, A.E., Marang, F., \& van Wyk, F. 1998b, MNRAS, 296, 317

Koen, C., O’Donoghue, D., Pollacco, D.L., \& Nitta, A. 1998c, MNRAS, 300, 1105

Morales-Rueda, L., Maxted, P.F.L, Marsh, T.R., North, R.C., \& Heber, U. 2002, MNRAS, in press (astro-ph/0209472)

O’Donoghue, D., Koen, C., Kilkenny, D., Stobie, R.S., \& Lynas-Gray, A.E. 1999, in 11th European Workshop on White Dwarfs, ed. J.-E. Solheim \& E.G. Meistas (PASPC), Vol. 169, 149

Orosz, J.A., \& Wade, R.A. 1999, MNRAS, 310, 773

Østensen, R., Heber, U., Silvotti, R., Solheim, J.-E., Dreizler, S., Edelmann, H. 2001b, A\&A, 378,466

Østensen, R., Solheim, J.-E., Heber, U., Silvotti, R., Dreizler, S., Edelmann, H. 2001a, A\&A, 368,175

Robinson, E.L., et al. 1995, ApJ, 438, 908

Saffer, R.A., Bergeron, P., Koester, D., \& Liebert, J. 1994, ApJ, 432, 351

Silvotti, R., Østensen, R., Heber, U., Solheim, J.-E., Dreizler, S., \& Altmann, M. 2002, A\&A, 383, 239

Wesemael, F., Allard, F., Lamontagne, R., Fontaine, G., \& Bergeron, P. 1997, in Third Conference on Faint Blue Stars, ed. A.G.D. Philip, J. Liebert, \& R.A. Saffer (Schenectady: Davis), 433

Wesemael, F., Fontaine, G., Bergeron, P., Lamontagne, R., \& Green, R.F. 1992, AJ, 104, 203 
Fig. 1.- The discovery light curve for PG1716+426 (top, observed with a V filter), followed by typical lightcurves for the largest amplitude long period pulsators: PG 1716+426 (R filter), PG 0850+170 (R), PG 1338+481 (B), PG 1627+017 (R), PB 5450 (R), PG 1739+489 (V). The time between peaks varies from about 35 to nearly 120 minutes.

Fig. 2.- The $\log g$ vs $T_{\text {eff }}$ diagram for various pulsating sdB stars superposed on Dorman's EHB evolutionary tracks (light dotted lines) for models with a range of $\mathrm{H}$ envelope masses. The thick $2 \sigma$ error bars represent the long period pulsators for which we have derived precise, homogeneous atmospheric parameters using high S/N MMT spectra. The stronger pulsators are plotted in red, and weaker pulsators in green. Thin black error bars show the nonpulsating sdB stars in our sample. The filled blue circles are the known short period EC 14026 pulsators, from a variety of sources (Charpinet 2001). The thicker dotted line represents the zero-age He-burning main sequence (i.e. the zero hydrogen envelope limit to the EHB). 


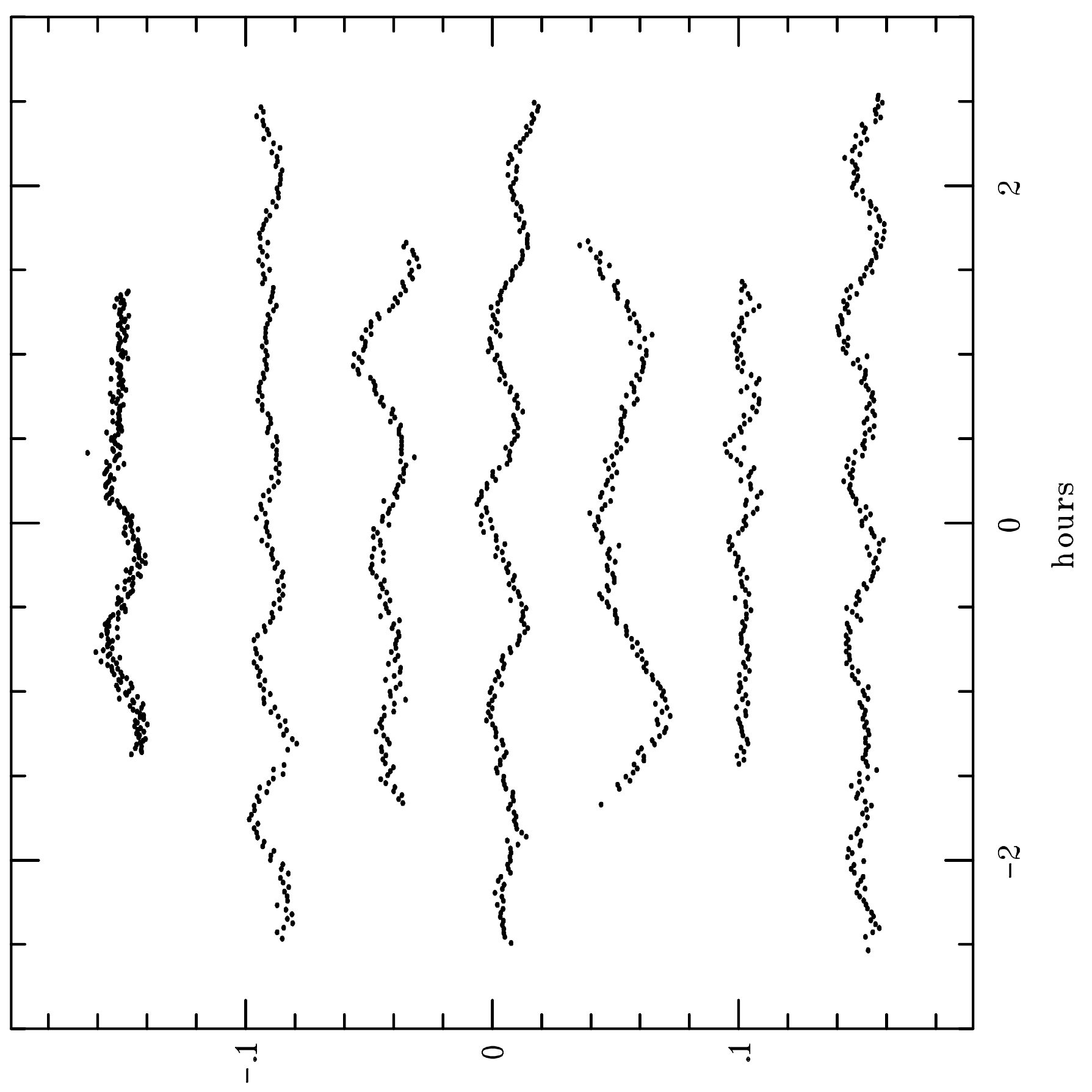

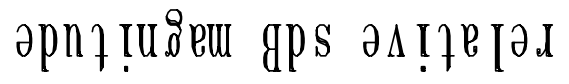




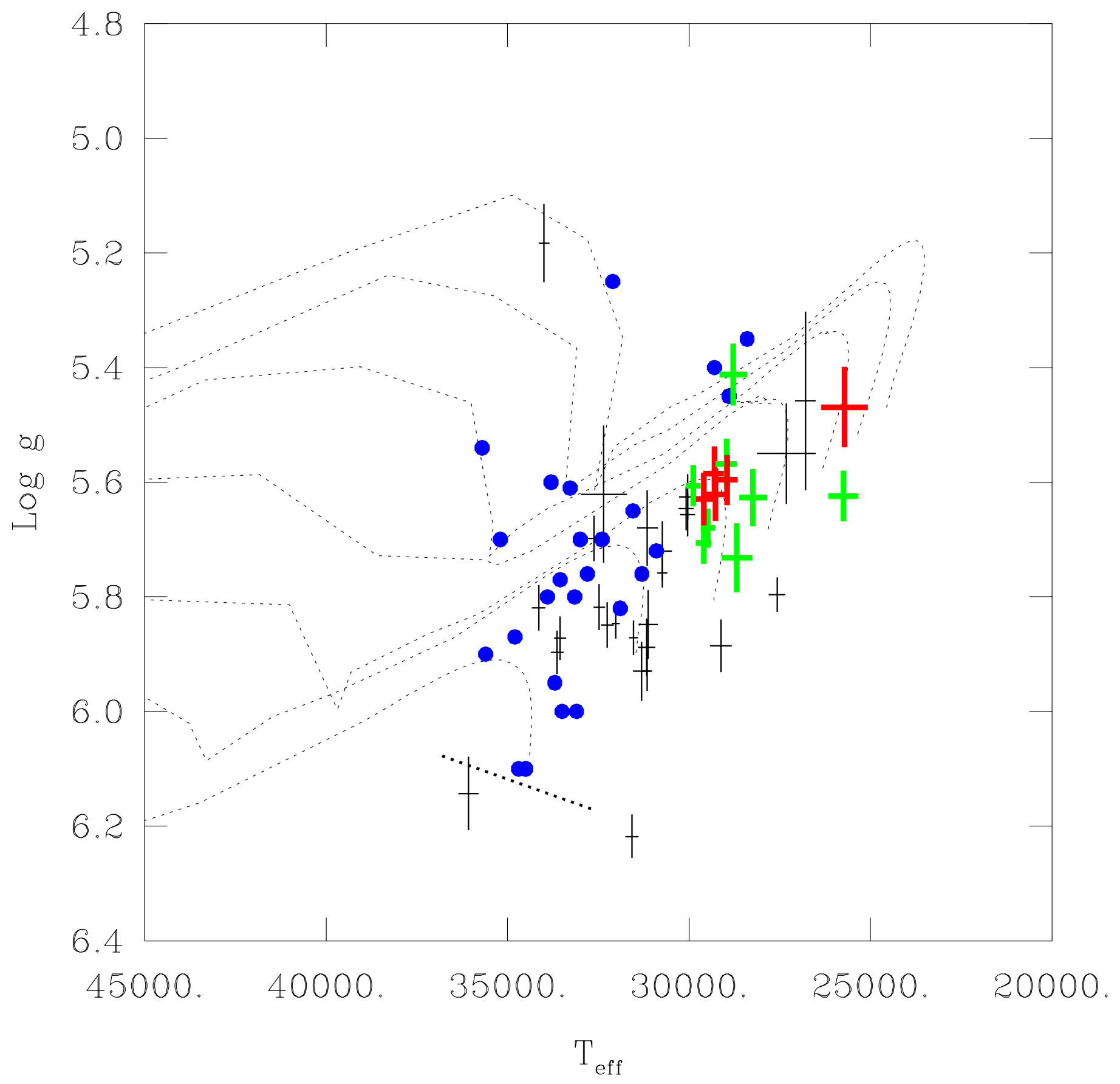

